\title{
Physician Event Reporting: Training the Next Generation of Physicians
}

\author{
Quang-Tuyen Nguyen, Joanna Weinberg, Lee H. Hilborne
}

\section{Abstract}

Physician reporting of adverse events and unsafe situations remains extremely low, despite the increased access to and use of electronic event reporting systems. We implemented an electronic, Web-based event reporting system at five University of California medical center campuses. While these campuses have witnessed approximately a three-fold increase in staff reporting following the implementation of the electronic system in 2003, physician reporting remains low-only 1.7 percent of all submitted reports were from physicians and only 4.5 percent of registered users are attending physicians and house officers. Our experience validates that of others; specifically, physician event reporting is a largely elusive practice. To change this behavior, it may be necessary to start at the beginning - to begin the discussion of error disclosure and adverse event reporting at the time when future physicians are still medical students. There have been several calls for medical schools and academic medical centers to improve patient safety and the quality of health care by explicitly educating and training the next generation of physicians in these areas. Although quality of care is implicit in most medical and other professional school curricula, medical students generally are not given the training necessary to meet the specific challenges outlined by the Institute of Medicine's Committee on Quality of Health Care in America reports, including To Err Is Human.

\section{Introduction}

According to the Institute of Medicine's (IOM) report in 2000, between 48,000 and 98,000 annual U.S. hospital deaths result from medical errors. ${ }^{1}$ Following the IOM report, there have been numerous calls to improve patient safety and place it at the forefront of the national agenda because these medical errors are often avoidable and the end result of a series of unnoticed antecedent events potentially involving multiple providers. ${ }^{2}$ To exacerbate the situation, our health care system is stressed, as evidenced by additional contributing factors like stress, burnout, and loss of autonomy among physicians; the reluctance of nurses and other health care personnel to challenge or question physicians' actions and orders; the complexity and interdependence of medical procedures; fear of litigation; and strained resources. Given the endemic nature of latent system errors in our current health care delivery system, it is no wonder that patient safety is compromised.

Aware that culture and tradition have a significant effect on framing how the health care community approaches quality and patient safety issues, the IOM 
recommends the creation of an open, honest, and blame-free culture as a preliminary first step toward providing better care. Specifically, individuals within the delivery system should not feel exposed or vulnerable when reporting a medical error or event - otherwise, disclosure will rarely occur, making it impossible to generalize trends or locate root causes of error. Low disclosure rates in comparison to the actual number of errors make it difficult to accurately assess the effectiveness and safety of the delivery system or subsequent interventions. Increasing reporting, however, requires building a supportive culture that acknowledges errors and encourages a proactive safety stance. Each adverse event or near miss should be viewed and used as a learning experience to better understand unsafe situations that can potentially lead to future unanticipated events.

There have been several calls for medical schools and academic medical centers to improve patient safety and the quality of health care by educating and training the next generation of physicians in these areas. ${ }^{3,4}$ Progressive medical colleges and academic medical centers with a strong commitment to improving the quality of health care delivery are well placed to champion a multifaceted patient safety program that teaches students the value of disclosure, event reporting, and the factors that contribute to errors. Because adverse events usually occur as a result of multiple failures, it is imperative that medical students learn to identify the multitude of factors that contribute to these events, so that they may foresee how to reduce the incidence of future events. 5,6

\section{Observations}

\section{Implementation of an electronic reporting system for staff use}

In May 2003, the Strategic Alliance for Error Reduction in California Healthcare (SAFER California Healthcare) saw the completion of an effort by the University of California health care campuses to implement an electronic, browser-based event reporting system for clinical use at the five academic medical centers and their associated institutions. One year later, the University of California Event Reporting System has successfully become an integral part of patient safety operations at all University of California health care campuses. Performance improvement, quality management, and risk management departments disclose evidence of types of reporting that originally had been nonexistent. Furthermore, reporting rates have increased overall. The medical centers are now finding it possible to track and analyze unsafe situations with a greater level of detail than before.

However, our campuses have also found that despite an easier, browser-based reporting system, physician event reporting remains extremely low. While the campuses have seen an average three-fold increase in staff report submissions, only 1.7 percent of all submitted reports are made by attending physicians and house officers, and only 4.5 percent of all registered users are attending physicians or house officers. The data reflect what previous research has shown- 
physician reporting is an elusive practice. It appears likely that event disclosure and patient safety discussion among physicians may also be rare. It would be unfair, however, to dismiss physicians as being uncooperative and unconcerned about patient safety; rather, the opposite is true. There are regular calls in the literature about how physician's need help and are unable to comfortably and appropriately address medical issues such as error. ${ }^{1}$ It is clear that issues regarding disclosure, error, and safety are important topics to address both now and in the future.

\section{Discussion}

\section{Traditional physician adverse event reporting behavior}

Physicians traditionally use a Morbidity \& Mortality (M\&M) conference format for reporting and discussing unsafe situations and adverse events, especially in academic teaching hospitals and schools where young trainees are taught. M\&M conferences are a way for medicine to self-regulate and for physicians to participate in their own quality review; they are universally viewed as "safe havens" because disclosed information remains protected and physicians are presumably able to discuss troubling near misses and adverse events to an assembly of understanding peers. Unfortunately, these conferences often fall short of their long-term implicit goals. Pierluissi and colleagues, observing traditional M\&M conferences, found that teachers had difficulty in explicitly acknowledging their medical error experiences. ${ }^{7}$ The sense of safety in conferences is often offset, in typical training program conferences, by the very public nature of the disclosure. Attending physicians are legally and socially responsible for the errors committed by resident trainees and medical students under their supervision; consequently a presentation in an M\&M can inadvertently result in a trainee feeling guilty, ashamed, and blamed by his superiors. Both the physician making the report and the audience participate in a mea culpa (my fault) analysis of the situation, which can be counterproductive to the larger goal of recognizing preventable latent system errors. ${ }^{2}$ The $M \& M$ conference format in itself is also not conducive for generalizing discussions to future situations. This is because discussions often center around the specifics of a particular case, missing the broader context of systematic contributing factors. Frequently, few quantitative data are presented with which to compare these incidences in the conferences. ${ }^{8,9}$ It is no wonder then, that despite the history and popularity of M\&M conferences in training centers, tackling the safety issue remains an ongoing challenge.

Except in cases of severe outcomes, physicians generally underuse institutional event reporting systems. This lack of a proactive stance places the physician in a passive position with respect to quality improvement. Resolution of unsafe circumstances may be perceived to be the responsibility of hospital risk management or performance improvement departments, not frontline clinical providers. Physician underuse of these patient safety tools, however, results in a less comprehensive understanding of the unsafe situations and factors leading up to 
some of the most frequent unanticipated outcomes. Event reporting, therefore, is biased because most reports are submitted by nursing and pharmacy personnel, so that reported events reflect issues most commonly encountered by and of concern to only these professionals. Issues of concern to physicians are often not captured.

\section{Current medical education is inadequate}

Current medical education has many responsibilities, the primary one being to teach students the content they must know to practice the art of medicine. Another main responsibility is to foster professionalism and leadership. In most U.S. medical schools, students spend their first two years learning the clinical and basic sciences, and their remaining two years integrating that body of information with supervised clinical experience. This curriculum is constantly improving to better educate future physicians. In particular, over the past decade, medical education has emphasized critical thinking among trainees, with many medical schools incorporating problem-based learning formats into their curriculum to foster these skills. ${ }^{10}$ The goal is to teach students not just the body of scientific knowledge, but also the relevant analytic skills and responsibilities necessary to be a good physician. Role-modeling with patients, mentoring through the clinical years, and discussion in a small group format have also become increasingly common.

This shift in education places physicians in a position to emulate the type of professionalism they need to demonstrate, both as leaders and as members of delivery teams. Research has shown, however, that most physicians do not know how to appropriately address the issue of medical mistakes. Some researchers have found physicians actually deal with these very sensitive issues in entirely dysfunctional ways, such as internalizing guilt, fear, anger, embarrassment and humiliation. ${ }^{11,12}$ To begin to address this issue, SAFER California Healthcare educators at UC Davis and UCLA developed a 2-minute video and discussion tool featuring a dialogue between a medical student, resident, intern, and an attending physician regarding a possible medical error and its disclosure to a patient or patient's family. Medical student feedback on the module has thus far been positive. The script was written to provoke discussion about the causes and frequency of error, how to approach patients and families following unexpected outcomes, and the importance of seeking support when errors occur to avoid the health care provider becoming a "second victim."12

Conventional training and practice in health care, especially in medicine, continue to attribute mistakes to failure on the part of the individual provider, using shame, blame, and fear techniques to mold students and residents and to reduce the likelihood of reoccurrence in future practice. This expectation reinforces a personal approach to responsibility and error in the health care setting, which inadvertently constructs barriers to examining and learning from a global and systems-based perspective. Because individual practitioners are taught to accept the blame for a mistake, they risk missing the deeper, possibly latent contributing factors, such as equipment, staffing, communication, and protocol design. Yet, since the shame and blame approach fails to resolve the error's 
source, errors will continue to happen. To reverse this trend, the importance of disclosing errors and unsafe situations must be instilled early in students' training. These future physicians must be explicitly taught that the occurrence of error is expected in the practice of medicine, and that errors are caused more often by latent systemic factors than by personal shortcomings. ${ }^{11-13}$

Physicians need to begin using quality tools in addition to the current M\&M conferences to discuss medical errors. At present, few training programs explicitly include patient safety and error evaluation in medical school, although the interest in such programs is increasing. Providing the appropriate instruments for practice would allow students to critically reflect on the different contributing factors that culminate in an unsafe situation. Critical skills gained from such practice would also give students a greater sense of awareness regarding these contributing factors and the ability to change the environment in which they practice. Such integration into the medical curriculum is timely, as there have been many calls to address safety and quality in medical school curricula, ${ }^{3}$ including one by the Association of American Medical Colleges stressing the value of continuous quality improvement. Numerous other educators, including Dr. Kenneth Shine, past president of the Institute of Medicine, are advocating a shift toward a paradigm that allows problem-solving for the $21^{\text {st }}$ century. ${ }^{14}$

\section{Recommendations}

\section{Target students to change the future}

One of the toughest challenges ahead for patient safety is changing culture and human behavior. The current lack of effective communication within the health care team is one of the leading causes for why errors are not caught before serious adverse events occur. These conditions can be ameliorated if physicians in training, as future team leaders, facilitate communication and encourage cultural change. ${ }^{15,16}$ Besides having greater collective power and organizing potential than other providers, physicians frequently have greater medical authority. Physicians, in fact, are responsible for coordinating most treatment and services received by the patient. This exposes the physician to greater liability, but also suggests that he or she should be in the best position to influence how care is provided, including the safety practices associated with such care. Physicians must assume responsibility for the function of the health care team, yet are underprepared to help create an environment supportive of the identification and amelioration of latent system errors. Culture change must begin by teaching physicians in training to appreciate how their current and future role on the provider team can be used to improve patient safety.

SAFER California Healthcare believes that implementing a blame-free, honest, and open reporting culture begins with interventions during medical education, before medical trainees begin serving as primary frontline health care providers. Any program that wishes to change the culture of medicine must address physicians-in-training, as well as current physicians. 
Using student insight into causes of near misses and unsafe situations during their clinical undergraduate years is an excellent way to target patient safety interventions. These medical students are ideal candidates for intervention because they are just beginning their clinical rotations and developing their professional habits. A student-focused event reporting system can reinforce their awareness of the frequency and causes of different medical errors they observe, while also allowing disclosure within a safe educational environment. Common findings reported by students should be integrated into discussions in courses that allow students to critically review cases and discuss key issues and contributing factors with mentors and peers.

Even though studies on unanticipated outcomes have not generally focused on trainee issues, most experts believe errors committed by medical students during their initial training years are common. ${ }^{17,18}$ Unfortunately, there are no data on how often medical students engage in, witness, or know of medical error-this situation needs to be rectified. Efforts to better prepare these trainees should be encouraged, and training-related data are especially important to guiding such efforts.

\section{Change how event reporting systems are used}

Traditionally, event reporting was a way to warn institutional administrators of possible litigation in the face of serious adverse events. Consequently, event reporting often gave a glimpse of the extreme and egregious types of medical errors, but an incomplete review of common latent errors contributing to these events. Effective reporting is limited by culture and tradition because many practitioners have had bad experiences when reporting events to superiors. ${ }^{19}$ Furthermore, staff are often confused as to which events to report and how to report them. ${ }^{20,21}$ Changing the stigma of event reporting and encouraging its practice among provider groups is key to capturing any tool's potential to affect safety and quality. ${ }^{22}$

Among students, event reporting systems should reinforce positive behavior and teach students the value of reporting. Systems can also help students and educators identify common training themes and concerns from which future interventions can be developed and refined. We believe these interventions will positively affect behavior in the future when students enter residency and, ultimately, practice.

\section{Ensure students understand contributing factors}

The current medical curriculum must be expanded to encourage reporting behavior and practice to develop a cadre of new graduates sensitive to patient safety issues and concerns. Ideally, a student-sensitive event reporting system will be unique from current systems in practice because it will not only capture unsafe events and circumstances, but also because it can, if properly designed, train students to recognize the deeper, underlying causes of these situations. An effective system that is also a teaching tool would allow students to enter reports of situations that they felt were unsafe and guide them through an analysis of the different contributing factors that could lead to an unsafe situation (e.g., personnel 
problems, vertical and horizontal communication flow, confusing procedure, inexperience, and equipment failure). Such a system would not only provide data on the types of errors or situations usually witnessed by medical students, but also generate reflection on and analysis of these situations among students and educators. This tool, combined with class seminars, will allow students to discuss uncomfortable situations much more openly.

\section{Design future interventions}

We may not yet be aware of the most common concerns students encounter regarding patient safety, either in terms of events they witness or uncomfortable situations they experience. Once those common themes are identified, case-based scenarios can be developed to facilitate students' understanding of how to approach such situations before encountering them in actual practice. Data collected from a student event reporting system will allow educators to develop modules that address situations and issues that are not adequately covered in existing curricula, such as interpersonal communication with professionals and patients, discomfort with the degree of student supervision, and responding constructively when errors occur. Discipline-specific themes that emerge can be addressed in individual student clerkships. Interventions can take the form of problem-based learning scenarios, greater role-modeling and role-playing experiences, and changes in the way our medical students are prepared for their clinical careers. This will allow students to increase their awareness about commonly encountered unsafe situations. ${ }^{23}$

\section{Conclusion}

Students graduating from schools with a patient safety curriculum that embraces error reporting should enter residency with a greater sensitivity for the subtler issues of patient safety and learn to realize that all adverse events are not the result of one mistake. They will learn that responsibility for safe care rests on understanding that faulty processes play a larger role than any singular act of commission or omission by any health care provider. The result will be a class of future physicians capable of investigating medical errors from a blame-free and nonjudgmental perspective, and who communicate better among themselves and with other providers on the team. Starting with students is a first step to shifting medicine toward a culture that is more open and communicative about the delivery of safe patient care.

\section{Acknowledgments}

Supported by a Developmental Center for Evaluation and Research in Patient Safety (DCERPS) grant no. HS-11512 from the Agency for Healthcare Policy and Research. 


\section{Author affiliations}

UCLA Center for Patient Safety and Quality, David Geffen School of Medicine at UCLA (LHH, QTN). Institute for Health and Aging, University of California, San Francisco (JW).

Address correspondence to: Lee H. Hilborne, M.D., M.P.H., UCLA Center for Patient Safety, David Geffen School of Medicine at UCLA, 10833 Le Conte Avenue, Los Angeles, CA 90095-1699; phone: 310 825-5656; e-mail: 1hilborne@mednet.ucla.edu or leeh@rand.org.

\section{References}

1. Kohn L, Corrigan J, Donaldson M, editors. To err is human: building a safer health system. A report of the Committee on Quality of Health Care in America. Institute of Medicine. Washington, DC: National Academy Press; 2000.

2. Rall M, Schaedle B, Zieger J, et al. Innovative training for enhancing patient safety. Safety culture and integrated concepts. Unfallchirurg 2002;105(11): 1033-42.

3. The Academic Medical Center Working Group of the Institute for Healthcare Improvement. The imperative for quality: a call for action to medical schools and teaching hospitals. Acad Med 2003;78(11):1085-9.

4. Greiner A, Knebel E. Health professions education: a bridge to quality. Washington, DC: National Academies Press, Inc.; 2003.

5. Pilpel D, Schor R, Benbassat J. Barriers to acceptance of medical errors: the case for a teaching program. Med Educ 1998;32(1):3-7.

6. Lester H, Tritter J. Medical error: a discussion of the medical construction of error and suggestions for reforms of medical education to decrease error. Med Educ 2001;35:855-61.

7. Pierluissi E, Fischer M, Campbell A, et al. Discussion of medical errors in morbidity and mortality conferences. JAMA 2003;290(21):2838-42.

8. Hamby L, Birkmeyer J, Birkmeyer C, et al. Using prospective outcomes data to improve morbidity and mortality conferences. Curr Surg 2000;57(4):384-8.

9. Feldman L, Barkum J, Barkum A, et al. Measuring postoperative complications in general surgery patients using an outcomes based strategy: comparison with complications presented at morbidity and mortality rounds. Surgery 1997;122(4):711-9.

10. Slavin S, Wilkes M, Usatine R. Faculty perceptions of learning while teaching in doctoring. Adv Health Sci Educ Theory Pract 1996;2(1):9-16.
11. Goldberg R, Kuhn G, Andrew L, et al. Coping with medical mistakes and errors in judgment. Ann Emerg Med 2002;39(3):287-92.

12. Wu A. Medical error: the second victim. BMJ 2000;320(7237):726-7.

13. An ethical dilemma: medical errors and medical culture. BMJ 2001;322(7296):1236-40.

14. Shine K. Health care quality and how to achieve it. Acad Med 2000;77(1):91-9.

15. Classen D, Kilbridge P. The roles and responsibility of physicians to improve patient safety within health care delivery systems. Acad Med 2002;77(10):963-72.

16. Shojania K, Wald H, Gross R. Understanding medical error and improving patient safety in the inpatient setting. Med Clin North Am 2002;86(4):847-67.

17. Supervising graduate medical students in the hospital. Jt Comm Perspect 2001;21(9):8,10.

18. Battles J, Shea C. A system for analyzing medical errors to improve GME curricula and programs. Acad Med 2001;76(2):125-33.

19. Lawton R, Parker D. Barriers to incident reporting in a healthcare system. Qual Saf Health Care 2002;11(1): $15-8$.

20. Wakefield B, Wakefield D, Uden-Holman T. Improving medication administration error reporting systems. Why do errors occur? Ambul Outreach 2000 (Spring):16-20.

21. Vincent C, Stanhope N, Crowley-Murphy M. Reasons for not reporting adverse incidents: an empirical study. J Eval Clin Pract 1999;5:13-21.

22. Dixon J. Going paperless with custom-built Webbased patient occurrence reporting. Jt Comm J Qual Improv 2002;28(7):387-95.

23. Epstein R. Mindful practice. JAMA 1999;282(9):833-9. 